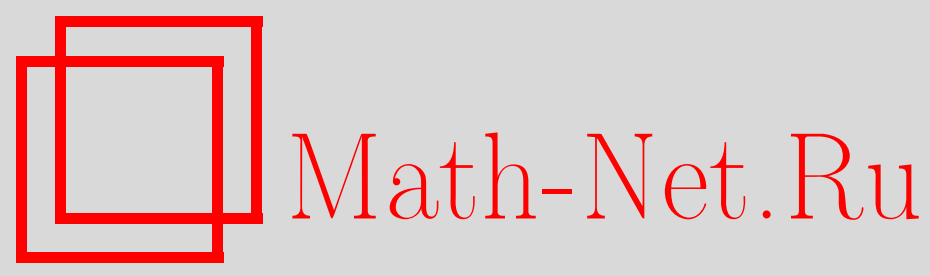

В. А. Романов, О неэквивалентности различных определений направлений дифференцируемости для векторных мер, Матем. заметки, 2002, том 72, выпуск 4, 528-534

DOI: https://doi.org/10.4213/mzm442

Использование Общероссийского математического портала Math-Net.Ru подразумевает, что вы прочитали и согласны с пользовательским соглашением http://www.mathnet.ru/rus/agreement

Параметры загрузки:

IP: 3.93 .64 .190

26 апреля 2023 г., 04:44:51

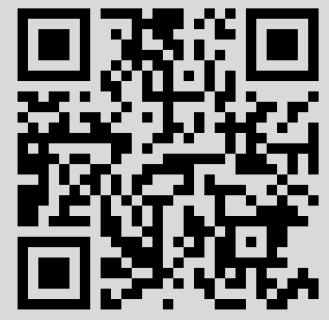




\title{
О НЕЭКВИВАЛЕНТНОСТИ \\ РАЗЛИЧНЫХ ОПРЕДЕЛЕНИЙ НАПРАВЛЕНИЙ ДИФФЕРЕНЦИРУЕМОСТИ ДЛЯ ВЕКТОРНЫХ МЕР
}

\section{В. А. Романов}

\begin{abstract}
В работе доказано, что для различных топологий - топологии сходимости на системе измеримьх множеств, топологии сходимости относительно полувариации и топологии сходимости по вариации - определения направлений дифференцируемости для векторных мер в общем случае попарно неэквивалентны, а также доказано, что для мер со значениями в банаховом пространстве, в котором выполняется свойство Радона-Никодима, соответствующие определения эквивалентны.

Библиография: 5 названий.
\end{abstract}

Известно [1], [2], что направления дифференцируемости относятся к наиболее существенным аспектам взаимосвязи между структурами меры и пространства. Для скалярных мер можно рассматривать определения дифференцируемости по направлению в трех топологиях (в порядке их усиления): в топологии $\tau_{f}$ слабой сходимости мер, в топологии $\tau_{s}$ сходимости на всех измеримых множествах и в топологии $\tau_{v}$ сходимости по вариации [1, с. 170], а для векторных мер естественно рассматривать определение дифференцируемости еще и в топологии $\tau_{s v}$ сходимости относительно полувариации (более сильной по сравнению с $\tau_{s}$, но более слабой по сравнению с $\left.\tau_{v}\right)$. Известно, что для скалярных мер определения дифференцируемости в топологиях $\tau_{s}$ и $\tau_{v}$ эквивалентны между собой $[1$, гл. 4 , теорема 2.1$]$, но являются строго более сильными по сравнению с дифференцируемостью в топологии $\tau_{f}[1$, с. 176]. Цель данной статьи - установление того факта, что для векторных мер в общем случае попарно неэквивалентными являются уже все четыре определения дифференцируемости, а также доказательство того, что для мер со значениями в банаховом пространстве со свойством Радона-Никодима определения дифференцируемости относительно топологий $\tau_{s}$ и $\tau_{v}$ (а поэтому и $\tau_{s v}$ ) остаются эквивалентными.

1. Обозначения и терминология. Пусть $Y$ - банахово пространство. Под $Y$-значными мерами понимаем счетно аддитивные функции множества конечной полной вариации, определенные на $\sigma$-алгебре всех борелевских подмножеств сепарабельного банахова пространства $X$ и принимающие значения в пространстве $Y$. Для меры $m$, вектоpa $h$ из $X$ и действительного числа $t$ под сдвинутой мерой $m_{t h}$ понимаем меру, задаваемую на измеримых подмножествах $E$ пространства $X$ формулой $m_{t h}(E)=m(E+t h)$. Пусть $\tau$ - одна из топологий $\tau_{s}, \tau_{s v}, \tau_{v}$ в пространстве $Y$-значных мер, определенных на $X$. 
В соответствии с [1], [2] мера $m$ назьвается дифференцируемой по направлению $h$ в топологии $\tau$, а мера $d_{h} m$ назьвается ее дифференциалом при приращении $h$, если в данной топологии имеет место равенство

$$
\lim _{t \rightarrow 0}\left(\frac{1}{t}\left(m_{t h}-m\right)-d_{h} m\right)=0
$$

В соответствии с [3] мера $m$ назьвается непрерывной по направлению $h$ ( $h$-непрерывной) в топологии $\tau$, если в этой топологии имеет место равенство

$$
\lim _{t \rightarrow 0}\left(m_{t h}-m\right)=0 .
$$

Если функция $f$ определена на подпространстве $L$ пространства $X$, а вектор $h$ принадлежит этому подпространству, то под функцией $f_{t h}$ понимаем функцию, задаваемую на элементах $x$ из $L$ формулой

$$
f_{t h}(x)=f(x+t h) .
$$

Через $v(m)$ обозначаем вариацию меры $m$. Значение вариации на всем пространстве $X$ обозначаем через $\operatorname{Var} m$, а значение полувариации на всем пространстве - чеpeз $\|m\|$.

Пусть $a$ и $b$ - два различных действительных числа и вектор $h$ пространства $X$ не равен нулю.

ОПРЕДЕЛЕНИЕ 1. T-функиией отрезка $D=[a h ; b h]$ пространства $X$ назьвается определенная на порожденном вектором $h$ одномерном подпространстве $L$ финитная функция с носителем $D$, значение которой в любой точке отрезка $D$ равно расстоянию от этой точки до ближайшего к ней конца отрезка.

ЗАмЕчАниЕ 1. Из определения $T$-функции сразу следует ее кусочная линейность, а также треугольньй вид ее подграфика (этим обстоятельством и объясняется введенное название), причем треугольник подг рафика равнобедренньй и прямоугольный, а его гипотенузой служит отрезок $D$.

ЗАмЕчАниЕ 2 . Поскольку $T$-функция отрезка $D$ абсолютно непрерьвна, а ее дифференщиал при приращении $h$ (существующий всюду, кроме трех точек - концов отрезка и его середины) суммируем, то из замечания 3.2.3 работы [2] вытекает, что скалярная мера, имеющая $T$-функцию своей плотностью, дифференцируема по направлению $h$ в топологиях $\tau_{s}$ и $\tau_{v}$.

\section{2. Вспомогательные результаты.}

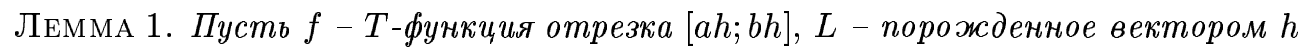
одномерное пространство. Тогда при выполнении условия $|t|<|b-a| / 2 \oint y н к-$ чия $g_{t}$, задаваемая на подпространстве $L$ формулой

$$
g_{t}(x)=\left|\frac{1}{t}\left(f_{t h}-f\right)(x)-d_{h} f(x)\right|,
$$

есть финитная функиия, ее носитель совпадает с обвединением трех имеющих одинаковую длину $|t| \cdot\|h\|$ отрезков:

$$
D_{1}=[(a-t) h ; a h], \quad D_{2}=\left[\left(\frac{b-a}{2}-t\right) h ; \frac{b-a}{2} h\right], \quad D_{3}=[(b-t) h ; b h],
$$

причем на отрезках $D_{1}$ и $D_{3}$ подграфик функиии $g_{t}$ имеет вид треугольников $c$ высотой $\|h\|$, а на отрезке $D_{2}$ подграфик имеет вид треугольника с высотой $2\|h\|$. 
ДокАЗАТЕЛЬСТвО. Носитель функции $f_{t h}$ получается из носителя функции $f$ сдвигом на вектор $(-t h)$. Поэтому носитель функции $g_{t}$ включается в объединение самого отрезка $D$ и его сдвига на указанньй вектор. Это объединение можно разбить на пять частей: на указанные в формулировке леммы отрезки $D_{1}, D_{2}, D_{3}$, а также на отрезки

$$
D_{4}=\left[a h ;\left(\frac{b-a}{2}-t\right) h\right], \quad D_{5}=\left[\frac{b-a}{2} h ;(b-t) h\right] .
$$

Ясно, что на каждом из отрезков $D_{4}$ и $D_{5}$ функции $f_{t h}$ и $f$ отличаются друг от друга на константу (свою для каждого из этих отрезков, но с общим модулем $|t| \cdot\|h\|)$. Ясно также, что функция $d_{h} f$ кусочно-постоянна и принимает (кроме нулевого) только два отличающихся знаком значения, модуль которых равен $\|h\|$, причем на каждом из отрезков $D_{4}$ и $D_{5}$ эти значения совпадают с соответствуюшими значениями функции $\left(f_{t h}-f\right) / t$. Поэтому из формулы (2) следует, что во всех точках отрезков $D_{4}$ и $D_{5}$ функция $g_{t}$ обращается в нуль. Что же касается подграфика функции $g_{t}$ на каждом из отрезков $D_{1}, D_{2}$ и $D_{3}$, то указанный в формулировке вид подграфика получается из кусочной линейности функций $f$ и $f_{t h}$ (на крайних отрезках $D_{1}$ и $D_{3}$ одна из них вообще обращается в нуль), а также из кусочного постоянства функции $d_{h} f$. Лемма доказана.

ЗАмЕчАнИЕ 3. Из леммы 1 следует, что при вьполнении условия $|t|<|b-a| / 2$ (т.е. когда величина $|t| \cdot\|h\|$ меньше половины длины носителя $T$-функции) площадь подграфика функции $g_{t}$ равна $2|t| \cdot\|h\|^{2}$.

ЗАмЕЧАнИЕ 4. Из определения $T$-функции легко следует, что для нее вьполняется неравенство

$$
|f(x+t h)-f(x)| \leqslant|t| \cdot\|h\| .
$$

С учетом того, что для дифференциала этой функции вьполняется неравенство

$$
\left|d_{h} f(x)\right| \leqslant\|h\|
$$

отсюда получаем, что для любых значений $t$ (уже без ограничения $|t|<|b-a| / 2$ ) все значения функции $g_{t}$, задаваемой формулой $(2)$, не превосходят числа $2\|h\|$.

Лемма 2. Пусть $Y$-значная мера $m$ непрерывна по направлению $h$ в топологии $\tau_{s}$. Тогда ее вариация $v(m)$ есть непрерывная по направлению $h$ скалярная мера.

ДокАЗАТЕЛЬСТво. Пусть $v_{1}-h$-непрерывная компонента в разложении вариации $v(m)$ в сумму $h$-непрерывной и вполне разрьвной мер [4, с. 65]. Тогда для любого функционала $y^{*}$ из единичного шара сопряженного к $Y$ пространства скалярная мера $y^{*} m$ $h$-непрерывна и мажорируется мерой $v_{1}$. Но тогда и сама векторная мера $m$ мажорируется мерой $v_{1}$. Следовательно, и вариация $v(m)$ мажорируется мерой $v_{1}$. Поэтому вариация $v(m)$ совпадает со своей $h$-непрерьвной компонентой $v_{1}$ и, следовательно, $h$-непрерывна. Лемма доказана.

Лемма 3. Пусть $Y$-значная мера $m$ дифференцируема по направлению $h$ в топологии $\tau_{s}$. Тогда мера $d_{h} m$ абсолютно непрерывна по мере $v(m)$. 
ДокАЗАТЕЛЬСТВо. Поскольку для каждого функционала $y^{*}$ из единичного шара сопряженного к $Y$ пространства скалярная мера $y^{*} m$ дифференцируема по направлению $h$ в топологии $\tau_{s}$, то из теоремы 2.6.5 работы [2] следует, что дифференциал этой скалярной меры при прирашении $h$ абсолютно непрерьвен по мере $v\left(y^{*} m\right)$, а поэтому и по мере $v(m)$. Следовательно, дифференциал векторной меры $m$ также абсолютно непрерьвен по мере $v(m)$. Лемма доказана.

\section{3. Основные результаты.}

Теорема 1. Пусть $Y=\ell_{\infty} u h$-ненулевой әлемент сепарабельного банахова пространства $X$. Тогда на б-алгебре всех борелевских подмнохсеств пространства $X$ существует такая $Y$-значная мера $m$, которая дифферениируема по направлению $h$ в топологии $\tau_{s v}$, но не дифференцируема по данному направлению в топологии $\tau_{v}$.

ДокАЗАТЕЛЬСтво. Пусть $L$ - одномерное подпространство, порожденное вектором $h$. Рассмотрим для каждого натурального $p \quad T$-функции отрезков

$$
\left[\frac{k-1}{p} h ; \frac{k}{p} h\right], \quad k=1, \ldots, p .
$$

Занумеруем все эти функции подряд в одну бесконечную последовательность $\left(f_{n}\right)$ и рассмотрим на $L$ скалярные меры $m_{n}$, плотности которых по одномерной мере Лебега совпадают с функциями $f_{n}$.

Заметим, что равномерно ограниченными являются как сами плотности $f_{n}$ (все они не превосходят половины длины отрезков, $T$-функциями которых служат), так и их дифференциалы $d_{h} f_{n}$ (модули последних не превосходят $\|h\|$ ). Поэтому из предложения 3 работы [3] вытекает, что $\ell_{\infty}$-значные функции множества $m$ и $d_{h} m$, компонентами которых служат скалярные меры на $L$ с плотностями соответственно $f_{n}$ и $d_{h} f_{n}$, являются $\ell_{\infty}$-значными мерами.

Для каждого действительного числа $t$ рассмотрим еще одну $\ell_{\infty}$-значную меру, a именно меру $\left(m_{t h}-m\right) / t-d_{h} m$. Применим к ней вторую часть того же предложения 3 работы [3]. В результате получаем равенства

$$
\begin{gathered}
\left\|\frac{1}{t}\left(m_{t h}-m\right)-d_{h} m\right\|=\sup _{n} \int_{L}\left|\frac{1}{t}\left(f_{n}(x+t h)-f_{n}(x)\right)-d_{h} f_{n}(x)\right| d x, \\
\operatorname{Var}\left(\frac{1}{t}\left(m_{t h}-m\right)-d_{h} m\right)=\int_{L} \sup _{n}\left|\frac{1}{t}\left(f_{n}(x+t h)-f_{n}(x)\right)-d_{h} f_{n}(x)\right| d x .
\end{gathered}
$$

Подьнтегральная функция в правой части равенства (3) отлична от нуля лишь на таком множестве, лебеговская мера которого не превосходит удвоенной длины носителя плотности $f_{n}$, а эта длина стремится к нулю при $n \rightarrow \infty$. Кроме того, в соответствии с замечанием 4 подьнтегральные функции равномерно ограничены по модулю (числом $2\|h\|$ ). Следовательно, для больших значений $n$ (начиная с некоторого $n_{0}$ ) интегралы в правой части равенства (3) можно сделать сколь угодно малыми равномерно относительно $t$. Для остальных значений $n$ (не превосходящих числа $n_{0}$ ) малость интегралов можно обеспечить за счет того, что $t \rightarrow 0$. Следовательно, при $t \rightarrow 0$ правая часть равенства (3) имеет нулевой предел. Поэтому в топологии $\tau_{s v}$ для векторной меры $m$ 
выполняется равенство (1), т.е. в этой топологии наша векторная мера дифференцируема по направлению $h$.

Осталось доказать отсутствие дифференцируемости векторной меры $m$ по направлению $h$ в топологии $\tau_{v}$.

С учетом того, что длины отрезков, по которым построены $T$-функции $f_{n}$, стремятся к нулю при $n \rightarrow \infty$, можно сделать вьвод о том, что при любом ненулевом фиксированном $t$ найдется натуральное число $n_{0}$ такое, что при вьполнении условия $n>n_{0}$ каждое значение функции $f_{n}$ по модулю не превосходит величину $|t| \cdot|| h \| / 4$. Поскольку модуль дифференциала $T$-функции при приращении $h$ принимает на своем носителе, совпадающем с носителем самой $T$-функции, значение $\|h\|$, то отсюда получаем, что при $n>n_{0}$ во всех точках носителя плотности $f_{n}$ значения функции, задаваемой формулой (2), не меньше величины $\|h\| / 2$. Поскольку объединение всех таких носителей (при всех $n>n_{0}$ ) совпадает с отрезком $[0 ; h]$, то отсюда следует, что во всех точках этого отрезка значения подьнтегральной функции из правой части равенства (4) не меньше положительной константы $\|h\| / 2$. Поэтому правая часть равенства (4) не стремится к нулю при $t \rightarrow 0$. Следовательно, в топологии $\tau_{v}$ для векторной меры $m$ равенство $(1)$ не выполняется, т.е. в указанной топологии наша векторная мера не дифференцируема по направлению $h$. Теорема доказана.

Теорема 2. Пусть $Y=\ell_{\infty} u h$-ненулевой элемент сепарабельного банахова пространства $X$. Тогда на б-алгебре всех борелевских подмнохсеств пространства $X$ существует такая $Y$-значная мера $m$, которая дифферениируема по направлению $h$ в топологии $\tau_{s}$, но не дифференцируема по данному направлению в топологии $\tau_{s v}$.

ДокАЗАТЕЛьСтво. Пусть, как и в доказательстве теоремы $1, L$ есть одномерное подпространство, порожденное вектором $h$. На этот раз для каждого натурального числа $n$ зададим плотность $f_{n}$ несколько иначе, а именно как сумму $n T$-функций отрезков

$$
\left[\frac{2 k}{2 n} h ; \frac{2 k+1}{2 n} h\right], \quad k=0,1, \ldots, n-1 .
$$

Эти плотности, как и их дифференциалы при приращении $h$, снова являются равномерно ограниченньми. Поэтому $\ell_{\infty}$-значные функции множества $m$ и $d_{h} m$, компонентами которых служат скалярные меры на $L$ с плотностями (относительно одномерной меры Лебега) соответственно $f_{n}$ и $d_{h} f_{n}$, суть $\ell_{\infty}$-значные меры.

Поскольку каждое измеримое подмножество одномерного подпространства $L$ можно сколь угодно точно в смысле меры симметрической разности апшроксимировать множествами, составленньми из конечного числа интервалов, то для доказательства дифоференцируемости векторной меры $m$ по направлению $h$ в топологии $\tau_{s}$ достаточно обосновать равенство

$$
\lim _{t \rightarrow 0} \sup _{n} \int_{E}\left(\frac{1}{t}\left(f_{n}(x+t h)-f_{n}(x)\right)-d_{h} f_{n}(x)\right) d x=0
$$

лишь для случая, когда множество $E$ является интервалом.

Для $T$-функции отрезка длины $\|h\| /(2 n)$ существует отрезок длины $(1 /(2 n)+|t|) \cdot\|h\|$, который включает в себя носитель как самой $T$-функции (а поэтому и носитель дифференциала этой функции), так и носитель ее сдвига на вектор (-th). Интеграл по 
этому отрезку от разности между самой $T$-функцией и ее сдвигом на вектор $(-t h), \mathrm{a}$ также интеграл от дифференциала функции при приращении $h$ равны нулю. С учетом этих обстоятельств и с учетом того, что плотность $f_{n}$ равна сумме $n T$-функций отрезков длины $\|h\| /(2 n)$, можно сделать вьвод о том, что для произвольного $\varepsilon>0$ существуют натуральное число $n_{0}$ и положительное число $\delta$ такие, что при вьполнении условий $n>n_{0}$ и $|t|<\delta$ найдутся $n$ отрезков (не обязательно дизьюнктных) длины $(1 /(2 n)+|t|) \cdot\|h\|$ каждый, объединение $A$ которых имеет следующие свойства: во-первых, лебеговская мера симметрической разности множеств $E$ и $A$ меньше $\varepsilon$ и, во-вторых, при замене множества $E$ в формуле (5) на множество $A$ соответствующий интеграл обратится в нуль. Поскольку подынтегральные функции в формуле (5) ограничены по модулю числом $2\|h\|$, то отсюда получаем, что при выполнении условий $n>n_{0}$ и $|t|<\delta$ модули интегралов, фигурирующих в формуле (5), меньше числа $2 \varepsilon \cdot\|h\|$. Для остальных значений $n$ (не превосходящих числа $n_{0}$ ) малость указанных интегралов можно обеспечить за счет того, что $t \rightarrow 0$. Следовательно, равенство (5) вьполняется.

Остается показать, что в топологии $\tau_{s v}$ построенная векторная мера $m$ не дифференцируема по направлению $h$.

Еще раз воспользуемся тем, что плотность $f_{n} n$-й компоненты $m_{n}$ нашей векторной меры равна сумме $n T$-функций отрезков длины $\|h\| /(2 n)$. Обозначим $t_{n}=1 /(6 n)$. Тогда величина $\left|t_{n}\right| \cdot\|h\|$ меньше половины длины упомянутых отрезков. Поэтому с учетом замечания 3 получаем, что для $n$-й компоненты выполняется равенство

$$
\left\|\frac{1}{t_{n}}\left(\left(m_{n}\right)_{t_{n} h}-m_{n}\right)-d_{h} m_{n}\right\|=\left(2\left|t_{n}\right| \cdot\|h\|^{2}\right) n=\frac{1}{3}\|h\|^{2} .
$$

Следовательно, для самой векторной меры вьполняется неравенство

$$
\left\|\frac{1}{t_{n}}\left(m_{t_{n} h}-m\right)-d_{h} m\right\| \geqslant \frac{1}{3}\|h\|^{2} .
$$

Поскольку правая часть этого неравенства не стремится к нулю, a $t_{n} \rightarrow 0$, то отсюда получаем, что в топологии $\tau_{s v}$ соотношение $(1)$ не выполняется, т.е. в указанной топологии векторная мера не дифференцируема по направлению $h$. Теорема доказана.

ЗАмЕчАнИЕ 5. Из построения плотностей $f_{n}$ в доказательствах теорем 1 и 2 нетрудно убедиться в том, что обе эти теоремы останутся в силе, если пространство $\ell_{\infty}$ ограниченных последовательностей заменить пространством $c_{0}$ сходящихся к нулю последовательностей.

ТеОрема 3. Пусть банахово пространство $Y$ обладает свойством Радона-Никодима, $h$ - ненулевой әлемент сепарабельного банахова пространства $X, m$ $Y$-значная мера, определенная на б-алгебре борелевских подмнохсеств пространства $X$ и дифференцируемая по направлению $h$ в топологии $\tau_{s}$. Тогда векторная мера $m$ дифференцируема по направлению $h$ и в топологии $\tau_{v}$.

ДокАЗАТЕЛьСтво. Поскольку векторная мера $m$ дифференцируема по направлению $h$ в топологии $\tau_{s}$, то она тем более непрерывна по направлению $h$. Согласно лемме 2 из непрерывности самой векторной меры вытекает непрерывность по данному направлению ее вариации $v(m)$, а из леммы 3 получаем, что дифференциал $d_{h} m$ абсолютно непрерьвен по мере $v(m)$. Поскольку пространство $Y$, в котором принимают значения как 
сама векторная мера $m$, так и ее дифференциал $d_{h} m$, имеет свойство Радона-Никодима, то отсюда вытекает, что дифференциал $d_{h} m$ можно представить как произведение некоторой интегрируемой по Бохнеру $Y$-значной функции и непрерьвной по направлению $h$ скалярной меры $v(m)$ с неотрищательньми значениями. Рассуждения, аналогичные доказательству теоремы 1 работы [5], показывают, что тогда дифференциал $d_{h} m$ нашей векторной меры непрерывен по направлению $h$ в топологии $\tau_{v}$. Следовательно, при $t \rightarrow 0$ стремится к нулю выражение в правой части неравенства

$$
\operatorname{Var}\left(\frac{1}{t}\left(m_{t h}-m\right)-d_{h} m\right) \leqslant \sup _{|s| \leqslant|t|} \operatorname{Var}\left(\left(d_{h} m\right)_{s h}-d_{h} m\right) \text {. }
$$

Это неравенство является аналогом соответствующего неравенства для скалярных мер $[1$, с. 171 , неравенство (2.4)] и доказьвается таким же способом. Но тогда при $t \rightarrow 0$ выражение в левой части неравенства тем более имеет нулевой предел. Это означает, что векторная мера $m$ дифференцируема по направлению $h$ в топологии $\tau_{v}$. Теорема доказана.

\section{СПИСОК ЦИТИРОВАННОЙ ЛИТЕРАТУРЫ}

[1] Далецкий Ю. Л., Фомин С. В. Меры и дифференциальные уравнения в бесконечномерых пространствах. М.: Наука, 1983.

[2] Авербух В.И., Смолянов О.Г., Фомин С.В. Обобщенные функции и дифференциальные уравнения в линейных пространствах. І. Дифференцируемые меры // Тр. ММО. 1971. T. 24. C. $133-174$.

[3] Романов В. А. О неэквивалентности трех определений непрерывных направлений для векторных мер // Матем. заметки. 1995. Т. 57. № 2. С. 310-312.

[4] Романов В.А. О разложении меры в линейном пространстве в сумму $H$-непрерьвной и вполне $H$-разрывной мер // Вестн. МГУ. Сер. 1. Матем., мех. 1976. Т. 31. № 4. С. 63-66.

[5] Романов В.А. Об $H$-непрерывных мерах в гильбертовом пространстве // Вестн. МГУ. Сер. 1. Матем., мех. 1977. Т. 32. № 1. С. 81-85. 